\title{
Resistance to high-fat-diet-induced obesity and sexual dimorphism in the metabolic responses of transgenic mice with moderate uncoupling protein 3 overexpression in glycolytic skeletal muscles
}

\author{
C. Tiraby $\cdot$ G. Tavernier $\cdot$ F. Capel $•$ A. Mairal $•$ \\ F. Crampes • J. Rami • C. Pujol • J. A. Boutin • \\ D. Langin
}

Received: 13 June 2007 / Accepted: 18 June 2007 / Published online: 4 August 2007

(C) Springer-Verlag 2007

\begin{abstract}
Aims/hypothesis Uncoupling protein (UCP) 3 is a mitochondrial inner membrane protein expressed predominantly in glycolytic skeletal muscles. Its role in vivo remains poorly understood. The aim of the present work was to produce a mouse model with moderate overproduction and proper fibre-type distribution of UCP3.

Methods Transgenic mice were created with a $16 \mathrm{~kb}$ region encompassing the human UCP3 gene. Mitochondrial uncoupling was investigated on permeabilised muscle fibres. Changes in body weight, adiposity and glucose or insulin tolerance were assessed in mice fed chow and high-
\end{abstract}

C. Tiraby and G. Tavernier contributed equally to this study.

C. Tiraby $\cdot$ G. Tavernier $(\bowtie) \cdot$ F. Capel $\cdot$ A. Mairal $\cdot$ F. Crampes $\cdot$

$\mathrm{J}$. Rami $\cdot$ C. Pujol $\cdot$ D. Langin

Inserm U858, Laboratoire de recherches sur les obésités,

Institut de Médecine Moléculaire de Rangueil,

Equipe 4, 1 avenue Jean Poulhès,

P.O. Box 84225, 31432 Toulouse Cedex 4, France

e-mail: getaver@toulouse.inserm.fr

C. Tiraby $\cdot$ G. Tavernier $\cdot$ F. Capel $\cdot$ A. Mairal $\cdot$ F. Crampes $\cdot$

J. Rami $\cdot$ C. Pujol $\cdot$ D. Langin

Institut Louis Bugnard, Université Paul Sabatier,

IFR31 Toulouse, France

D. Langin

CHU de Toulouse, Laboratoire de biochimie,

Institut Fédératif de Biologie de Purpan,

Toulouse, France

\section{J. A. Boutin}

Division de Pharmacologie Moléculaire et Cellulaire,

Institut de Recherches Servier,

Croissy-sur-Seine, France fat diets. Indirect calorimetry was used to determine wholebody energy expenditure and substrate utilisation.

Results Transgenic mice showed a twofold increase in UCP3 protein levels specifically in glycolytic muscles. Mitochondrial respiration revealed an increase of uncoupling in glycolytic but not in oxidative muscles. Transgenic mice gained less weight than wild-type littermates due to lower adipose tissue accretion when fed a high-fat diet. Animals showed a sexual dimorphism in metabolic responses. Female transgenic mice were more glucose-sensitive than wild-type animals, while male transgenic mice with high body weights had impaired glucose and insulin tolerance. Measurements of RQs in mice fed chow and high-fat diets suggested an impairment of metabolic flexibility in transgenic male mice. Conclusions/interpretation Our data show that physiological overproduction of UCP3 in glycolytic muscles results in mitochondrial uncoupling, resistance to high-fat dietinduced obesity and sex specificity regarding insulin sensitivity and whole-body substrate utilisation.

Keywords Glucose tolerance - Glycolytic skeletal muscle . High-fat diet · Metabolic flexibility · Mouse - Obesity ·

Sexual dimorphism $\cdot$ UCP3 $\cdot$ Uncoupling

$\begin{array}{ll}\text { Abbreviations } \\ \text { FCCP } & p \text {-trifluoromethoxy-carbonyl-cyanide-phenyl } \\ & \text { hydrazone } \\ \mathrm{h} & \text { human } \\ \mathrm{m} & \text { mouse } \\ \mathrm{UCP} & \text { uncoupling protein } \\ V \mathrm{CO}_{2} & \text { carbon dioxide production } \\ V_{2} & \text { oxygen consumption } \\ \mathrm{WT} & \text { wild-type }\end{array}$




\section{Introduction}

Obesity and type 2 diabetes are the most common metabolic diseases in the world. The two conditions are rapidly becoming a global pandemic, causing great concern. Insulin resistance constitutes a critical link between the excess fat mass seen in obesity and the development of type 2 diabetes. Mitochondrial dysfunction appears to be an essential component in the development of skeletal muscle insulin resistance [1]. In that respect, skeletal muscle-specific elements of the mitochondrion may play an important role in the regulation of insulin sensitivity and fatty acid metabolism.

Uncoupling protein (UCP) 3 is a protein of the mitochondrial membrane predominantly produced in skeletal muscle in humans [2-4]. The UCP3 gene encodes a protein with sequence similarities to other members of the UCP family. The uncoupling activity of these proteins is a consequence of their protonophoric activity, which leads to dissipation of the electrochemical gradient established by the respiratory chain. UCP3 is the only member of the family with a tissue distribution restricted to skeletal muscle in adult humans. UCP3 levels have been reported to be higher in type II fast glycolytic and glycolytic-oxidative fibres than in type I slow oxidative fibres [5]. Based on the location and putative role in proton and/or anion transport, several functions have been proposed for UCP3. By analogy with UCP1, UCP3 may play a role in thermogenesis. The phenotype of Ucp3 knockout mice and data obtained in hibernators argues against thermogenesis being the major function of UCP3 [6-8]. However, UCP3 may influence thermogenesis under certain circumstances [9]. Another potential role is protection against damage caused by excess reactive oxygen species production, which is known to contribute to hyperglycaemia-induced damage in muscle [10, 11]. Accordingly, mice lacking UCP3 show evidence of increased reactive oxygen species production and oxidative damage in skeletal muscle mitochondria [6, 12]. UCP3 may also play a role in the transport of fatty acid anions. In one model, UCP3 prevented accumulation of non-esterified fatty acids within the mitochondria, preventing mitochondrial damage [13]. In a second model, the export of fatty acyl anions allowed fatty acid reactivation by acyl-CoA synthetases to support fatty acid oxidation $[14,15]$. Other types of ion transport, which would ensure fine tuning of the exchanges between glycolysis and oxidative phosphorylation, have recently been proposed to be mediated by UCP3 [16]. A role in the crosstalk between glucose and fatty acid metabolism is suggested by the effect of UCP3 overexpression in human muscle cells. It results in an increase of both fatty acid and glucose oxidation, but also in an enhancement of fatty acid-mediated inhibition of glucose oxidation [17]. These data suggest a role for UCP3 in nutrient partitioning.
UCP3 is present in fully differentiated skeletal muscle cells [18]. Cultured muscle cells produce little if any UCP3 and consequently do not constitute an appropriate cellular context for the study of UCP3 functions. The purpose of this study was to investigate the impact of moderate UCP3 overproduction on mouse energy balance and insulin sensitivity in vivo. We produced transgenic mice producing human UCP3 (h-UCP3) under the control of its own promoter. This strategy ensured moderate UCP3 overproduction specifically targeting type II skeletal muscles. Therefore, the UCP3 distribution seen in human tissues and the physiological range of variations observed in clinical studies were respected [5, 19-21]. Mitochondrial uncoupling was investigated on permeabilised muscle fibres. Changes in body weight, adiposity and glucose or insulin tolerance were assessed in mice fed chow and high-fat diets. Indirect calorimetry was used to determine whole-body energy expenditure and substrate utilisation. Experiments were performed both in female and male animals to assess putative sex effects in metabolic responses.

\section{Methods}

Transgenesis DNA was extracted from a bacterial artificial chromosome clone (Genbank accession number AF050113). We amplified $5 \mathrm{ng}$ by PCR (Expand Long Template PCR System; Roche Diagnostics, Meylan, France) and the following primers: 5' GGCCTCCCAAAGTGCTGGGGT TACAGA $3^{\prime}$ and 5' TGCACACCACATGTCTGCTTTC CAAGG 3'. The 16023 bp product was subcloned into the pGEMT easy cloning vector (Promega, Charbonnières, France) and sequenced using a kit (Big Dye Terminator v3.1; Applied Biosystems, Courtaboeuf, France). It was digested with NotI to obtain a linearised fragment ready to inject into one-cell embryos.

Animals Animal studies followed the INSERM and Louis Bugnard Institute Animal Core Facility guidelines. All animals were purchased from Elevage Janvier (Le Genest St Isle, France). B6D2/F1 female mice were hormonally treated and mated with B6D2/F1 male mice as previously described [22]. Putative founders were screened by PCR from tail DNA. To estimate transgene copy number, genomic DNA from F1 mice was subjected to Southern blotting as previously described [23]. In the different studies, transgenic mice were compared with wild-type (WT) littermates of the sixth to eighth generation on the B6D2 background. Animals were raised with alternating 12 h light-dark cycles (lights on 07:00-19:00 hours).

Diets After weaning, WT and h-UCP3 mice were fed either a chow diet or a high-fat diet (45\% energy as fat, $35 \%$ as 
carbohydrate and $20 \%$ as protein; Safe, Augy, France). Each animal was weighed weekly. Food intake was measured daily only in animals fed a chow diet and housed individually [24]. Mice were killed at 27 weeks of age, blood was collected in heparinised tubes and various tissues were removed, immediately weighed, frozen in liquid nitrogen and stored at $-80^{\circ} \mathrm{C}$. The length of the femur was measured as an index of growth.

Protein analysis Mitochondria were prepared from gastrocnemius and soleus muscles as previously described [25]. Electrophoresis of $10 \mu \mathrm{g}$ of mitochondrial proteins was performed on a $12 \%$ SDS-PAGE gel; proteins were then transferred on to nitrocellulose membranes (Hybond ECL; GE Healthcare, Orsay, France). Membranes were incubated with an antibody raised against h-UCP3 (Antibody CabrX; Research Diagnostics, San Antonio, TX, USA). The antibody has been validated for detection of both h-UCP3 and mouse UCP3 (m-UCP3) [25]. A single amino acid is different between human and mice in the 14 amino acid peptide used to raise the antibody. However, small differences in cross-reactivity between the two species cannot be ruled out. Revelation was performed using enhanced chemiluminescence and quantified with ImageQUANT 1.5 software (GE Healthcare). To normalise the results, membranes were dehybridised and hybridised again with a purified mouse anti-cytochrome $c$ antibody (BD Biosciences, Le Pont de Claix, France).

mRNA analysis Total RNA from various tissues was isolated using RNASTAT-60 (AMS Biotechnology, Abingdon, UK) or RNeasy kit (Qiagen, Courtaboeuf, France) for adipose tissues. Total RNA $(1 \mu \mathrm{g})$ was treated with DNase I then retrotranscribed using random hexamers and Thermoscript reverse transcriptase (Invitrogen, Cergy, France) to measure gene expression. Real-time quantitative PCR was performed on GeneAmp 7000 sequence detection system (Applied Biosystems) using Sybr green chemistry. Primers were designed to amplify selectively $\mathrm{m}-U c p 3$ and $\mathrm{h}-U C P 3$ or the UCP3 transcripts from both species (hm-UCP3) using Primer Express 1.5 (Applied Biosystems). 18S rRNA was used as control to normalise gene expression using an assay kit (rRNA Control Taqman; Applied Biosystems).

Oxygen consumption in permeabilised fibres Mitochondrial oxygen consumption $\left(V_{2}\right)$ was determined in freshly permeabilised fibres from soleus and tibialis anterior muscles. Briefly, 10-20 mg of each muscle were dissected in ice-cold buffer A containing: $2.8 \mathrm{mmol} / 1 \mathrm{CaK}_{2}$ EGTA, $7.2 \mathrm{mmol} / 1 \mathrm{~K}_{2}$ EGTA, $20 \mathrm{mmol} / 1$ imidazole, $0.5 \mathrm{mmol} / \mathrm{l}$ DTT, $6.6 \mathrm{mmol} / \mathrm{l} \mathrm{MgCl} 2,50 \mathrm{mmol} / 1$ 4-morpholineethanesulphonic acid (MES), $20 \mathrm{mmol} / \mathrm{l}$ taurine, $5.8 \mathrm{mmol} / \mathrm{l}$ ATP,
$20 \mathrm{mmol} / \mathrm{l}$ phosphocreatine and $0.2 \%$ BSA (pH 7.1). Fibres were gently separated using a pair of needles and forceps under a microscope. Bundles of $8-10 \mathrm{mg}$ of fibres were permeabilised for $25 \mathrm{~min}$ at $4{ }^{\circ} \mathrm{C}$ under permanent platerotator agitation in ice-cold buffer A containing $60 \mu \mathrm{g} / \mathrm{ml}$ of saponin. Three washings of $10 \mathrm{~min}$ were performed in buffer B, containing: $2.8 \mathrm{mmol} / 1 \mathrm{CaK}_{2}$ EGTA, $7.2 \mathrm{mmol} / 1$ $\mathrm{K}_{2}$ EGTA, $20 \mathrm{mmol} / 1$ imidazole, $0.5 \mathrm{mmol} / 1 \mathrm{DTT}$, $3 \mathrm{mmol} / 1 \mathrm{KH}_{2} \mathrm{PO}_{4}, 4.2 \mathrm{mmol} / 1 \mathrm{MgCl}_{2}, 110 \mathrm{mmol} / 1 \mathrm{MES}$, $20 \mathrm{mmol} / 1$ taurine and $0.2 \% \mathrm{BSA}(\mathrm{pH} 7.1)$. Fibres were then stored in buffer $\mathrm{B}$ under permanent plate-rotator agitation until respiration measurements. Respiratory measurements were performed using a Clark-type oxygen electrode (Hansatech Instruments, Norfolk, UK) under permanent stirring at $25^{\circ} \mathrm{C}$ in buffer B. Maximal $V \mathrm{O}_{2}$ was determined in the presence of glutamate $(5 \mathrm{mmol} / \mathrm{l})$-malate $(2 \mathrm{mmol} / \mathrm{l})$ and $500 \mu \mathrm{mol} / \mathrm{l}$ ADP. Successive additions of oligomycin $(10 \mu \mathrm{g} / \mathrm{ml})$ and $1 \mu \mathrm{mol} / \mathrm{l} p$-trifluoromethoxycarbonyl-cyanide-phenyl hydrazone (FCCP) were performed to inhibit ATP synthase and uncouple oxygen consumption from ATP synthesis, respectively. Bundles of fibres were retrieved and dried at room temperature for $48 \mathrm{~h}$ to estimate net dry weight.

Glucose and insulin tolerance tests Mice were fasted either overnight or for $6 \mathrm{~h}$ with free access to drinking water. Baseline glucose level was recorded on tail blood using a one-touch basic glucometer (Roche Diagnostics, Meylan, France). Glucose was administered either intraperitoneally $(5.6 \mu \mathrm{mol} / \mathrm{g})$ or by oral gavage $(8.4 \mu \mathrm{mol} / \mathrm{g})$ and blood glucose was measured at $10,20,30,40,60,90$ and $120 \mathrm{~min}$ after glucose administration. Oral and intraperitoneal glucose administration gave similar results. For insulin tolerance tests, insulin was administered intraperitoneally $(1 \mathrm{mU} / \mathrm{g})$ and blood glucose was measured $15,30,45,60$, 90 and 120 min after injection.

Indirect calorimetry $V \mathrm{O}_{2}$ and carbon dioxide production $\left(V \mathrm{CO}_{2}\right)$ of $\mathrm{h}-U C P 3$ and WT mice were measured using a four-chamber oxylet system (Bioseb, Chaville, France). Temperature was maintained at $22^{\circ} \mathrm{C}$ and the light was on from 07:00 to 19:00 hours. System settings included a flow-rate of $0.6 \mathrm{1} / \mathrm{min}$, a sample purge of $5 \mathrm{~min}$ and a measurement period of $5 \mathrm{~min}$ every $25 \mathrm{~min}$. Twenty-four hours prior to data collection, mice were placed in separate calorimetry chambers (each with a volume of 2.51 ), with free access to food and water. The RQ was calculated as the ratio of $V \mathrm{CO}_{2}: V \mathrm{O}_{2}$; results were expressed as per cent relative cumulative frequency along the measurement period [26]. Control experiments were performed in fasting conditions. As expected, $V \mathrm{O}_{2}$ and $\mathrm{RQ}$ were statistically significantly increased and decreased respectively during fasting in male and female mice (data not shown). 
Statistical analyses A Mann-Whitney non-parametric statistical test was applied to compare food intake, adiposity and mitochondrial oxygen consumption between control and transgenic animals. ANOVA tests were performed to compare increases in body weight and glucose levels from the various in vivo tests. Data analyses were performed using SPSS version 12.0 for Windows (SPSS, Chicago, IL, USA).

\section{Results}

Expression of human UCP3 in transgenic mice The $16 \mathrm{~kb}$ transgene contained $6.2 \mathrm{~kb}$ of the promoter region, $9.3 \mathrm{~kb}$ encompassing exons and introns and $0.5 \mathrm{~kb}$ of the $3^{\prime}$ region of the h-UCP3 gene (Fig. 1). Following microinjection into fertilised eggs, three founders were obtained. Two lines, A and $\mathrm{B}$, were established on the $\mathrm{B} 6 \mathrm{D} 2$ genetic background with 20 and 10 copies of the transgene integrated into genomic DNA, respectively. The $\mathrm{h}-U C P 3$ expression profile determined by RT-PCR was similar to that observed in humans, i.e. skeletal muscle $>$ heart $\geq$ brown adipose tissue (Fig. 2a,b). Furthermore, skeletal muscle h-UCP3 expression could be subclassified as follows according to fibre types: type IIB/glycolytic (biceps femoris) $\geq$ type IIA and IIB/glycolytic-oxidative (gastrocnemius)>type IIX/ oxidative (diaphragm) $>$ type I/oxidative (soleus). As expected, liver, spleen and brown adipose tissue did not express $\mathrm{h}-U C P 3$. The $\mathrm{h}-U C P 3 \mathrm{mRNA}$ profile was similar to that of $\mathrm{m}-U c p 3$ except that a higher expression of $\mathrm{m}$ Ucp3 was observed in brown adipose tissue and heart (Fig. 2c). All subsequent analyses were performed on transgenic line B. Using primers that do not discriminate between $\mathrm{h}-U C P 3$ and $\mathrm{m}-U c p 3, U C P 3$ mRNA overexpression was seen in skeletal muscles with type IIA and type IIB fibres but not in soleus muscle and other tissues (Fig. 2d). Male and female mice gave similar results. UCP3 protein was quantified in mitochondria isolated from gastrocnemius and soleus of $\mathrm{h}-U C P 3$ and WT mice (Fig. 3). In the gastrocnemius mitochondria, UCP3 protein levels in male and female transgenic mice were double those of WT animals (2.4 \pm 0.1 -fold and 2.2 \pm 0.1 -fold, $n=4$ in each group). No difference in UCP3 level was observed in the soleus muscle.

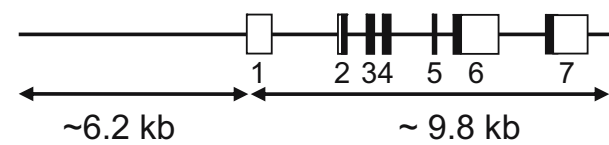

Fig. 1 Structure of the h-UCP3 transgene. Boxes and lines represent exons labelled from 1 to 7 and introns, respectively. Closed and open boxes represent coding and non-coding exons. Values in $\mathrm{kb}$ : approximative lengths of the various regions
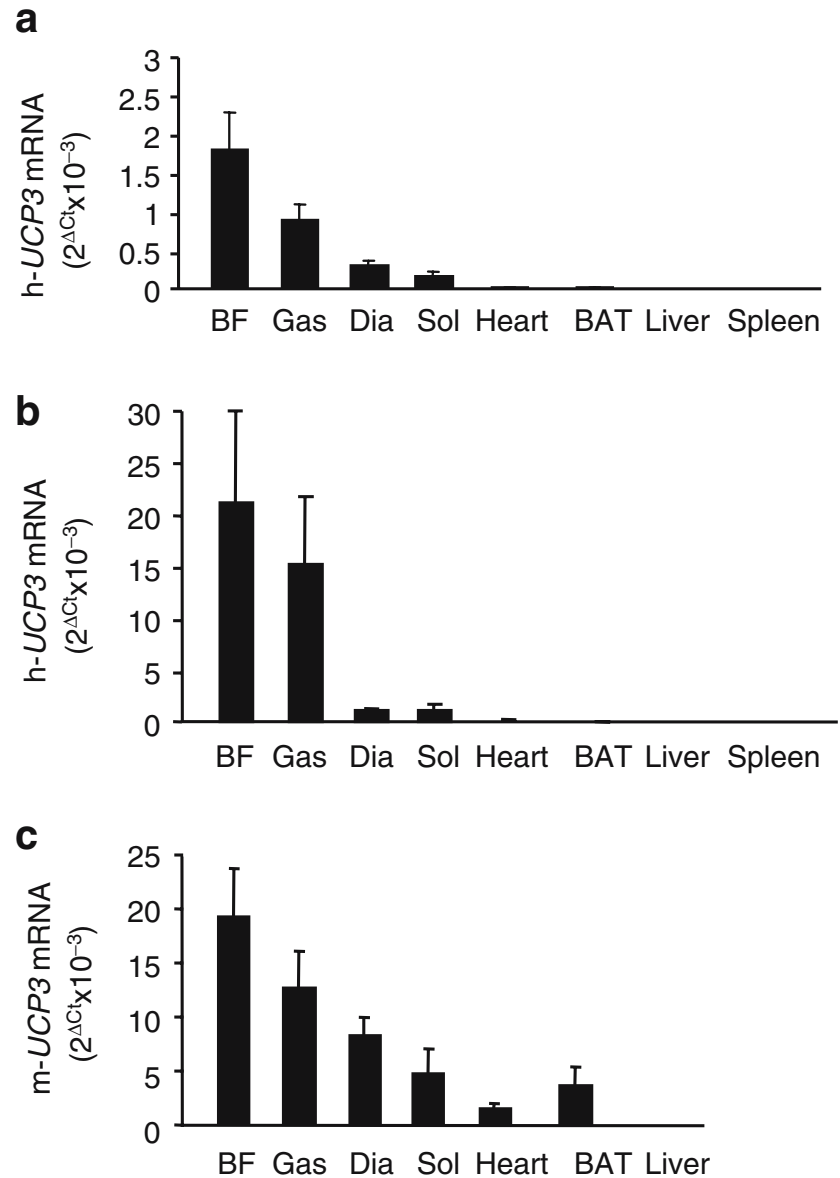

d

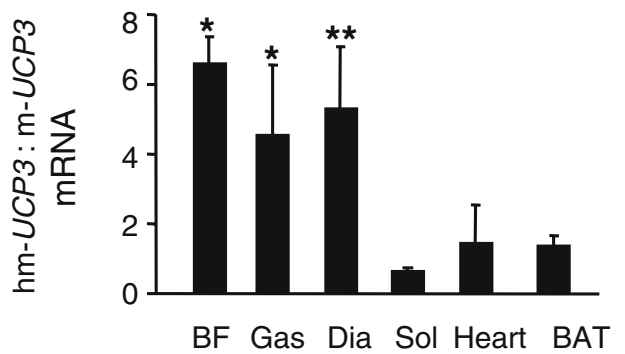

Fig. 2 Expression of human and mouse $U C P 3$ mRNA in transgenic mice. $\mathbf{a}, \mathbf{b}$ h- $U C P 3$ gene expression in tissues of transgenic line $\mathrm{A}(n=9)$ and transgenic line $\mathrm{B}(n=10)$, respectively. $\mathbf{c} \mathrm{m}-U c p 3$ expression in tissues of transgenic line B $(n=12)$. $\mathbf{d}$ Ratio between human and mouse $U C P 3$ expression $(\mathrm{hm}-U C P 3)$ and $\mathrm{m}-U c p 3$ in tissues of transgenic line $\mathrm{B}(n=6)$. mRNA levels were quantified by RT real-time PCR. Data are expressed as $2^{\Delta \mathrm{Ct}} \times 10^{-3}$ with $18 \mathrm{~S}$ RNA used to normalise the values. $B F$, biceps femoris; Gas, gastrocnemius; Dia, diaphragm; Sol, soleus; $B A T$, brown adipose tissue. ${ }^{*} p<0.05 ;{ }^{*} p<0.01$

Effect of UCP3 overexpression on $\mathrm{VO}_{2}$ in permeabilised fibres We wished to determine whether UCP3 overexpression had an effect on mitochondrial respiration. We therefore compared $V \mathrm{O}_{2}$ in permeabilised fibres from the glycolytic tibialis anterior muscle with that from the oxidative soleus muscle, which showed no overexpression 


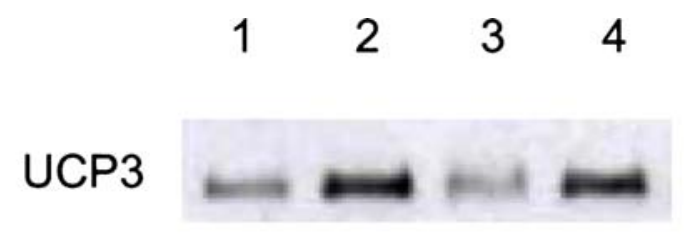

\section{Cytochrome $c$}

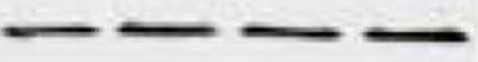

Fig. 3 UCP3 levels in mitochondria of the gastrocnemius muscle. Lanes 1 and 3, WT mice; lanes 2 and 4, h-UCP3 mice

of UCP3. The respiratory rate upon addition of ADP was similar for the two genotypes in the tibialis anterior and soleus muscles (Fig. 4). After addition of oligomycin, an inhibitor of mitchondrial ATP synthesis, respiration was significantly higher in the tibialis anterior muscles of h-UCP3 mice than in WT littermates. Addition of the chemical uncoupling agent FCCP led to similar respiration in the two genotypes. No difference was observed in soleus muscles of h-UCP3 and WT mice.

Evolution of body weight and body composition in h-UCP3 mice fed chow or high-fat diets On a chow diet, curves of body weight were similar between $\mathrm{WT}$ and $\mathrm{h}-U C P 3$ mice (Fig. 5). WT mice fed a high-fat diet showed a greater
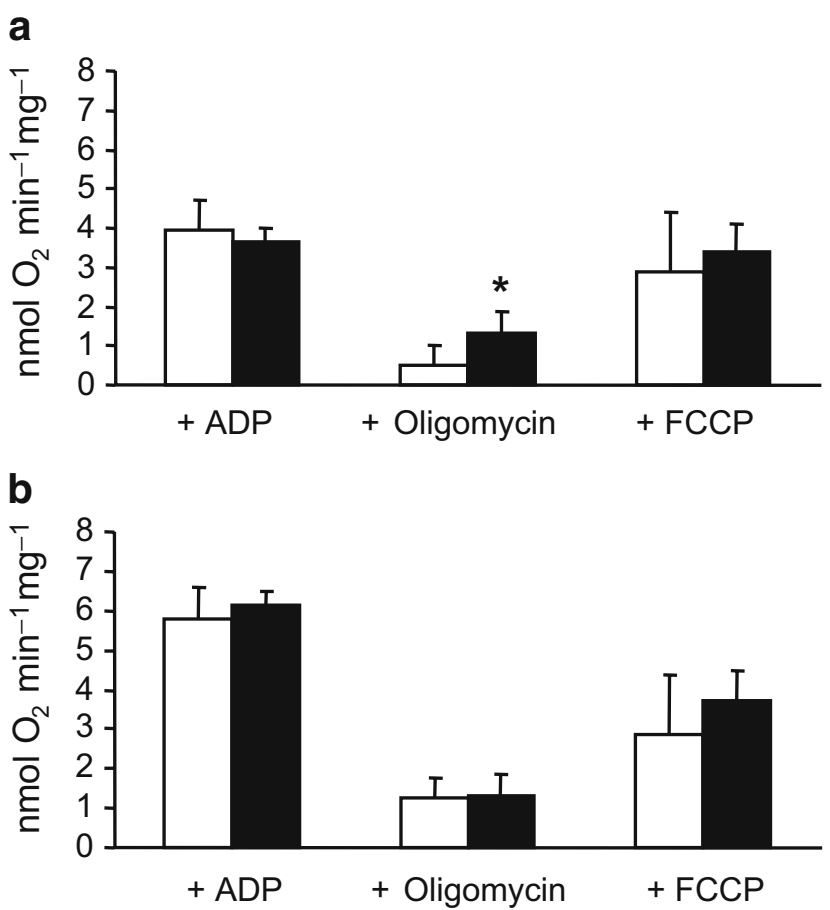

Fig. 4 Mitochondrial $V \mathrm{O}_{2}$ in permeabilised skeletal muscle fibres. a Respiration of tibialis anterior fibres and $\mathbf{b}$ of soleus fibres expressed in nmol $\mathrm{O}_{2}$ consumed per min and per mg of dry muscle. ${ }^{*} p<0.05$ between h-UCP3 (closed bars) and WT (open bars) male mice $(n=6)$ using a Mann-Whitney non parametrical statistical test

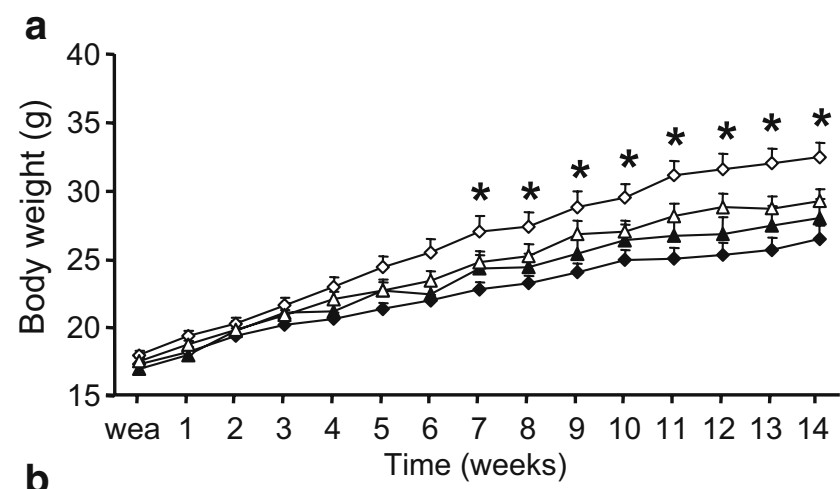

b

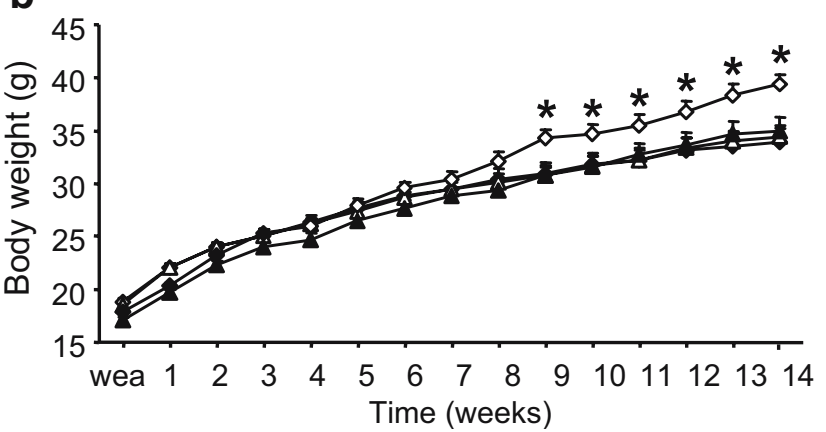

Fig. 5 Body weight curves of female (a) and male (b) mice from weaning (wea) to week 14 of diet. a closed diamonds, female mice WT fed a chow diet, $n=26$; closed triangles, h-UCP3 mice fed a chow diet, $n=27$; open diamonds, WT fed a high-fat diet, $n=23$; open triangles, h-UCP3 fed a high-fat diet, $n=27$. b closed diamonds, male WT mice fed a chow diet, $n=22$; closed triangles, h-UCP3 mice fed a chow diet, $n=22$; open diamonds, WT fed a high-fat diet, $n=35$; open triangles, h-UCP3 mice fed a high-fat diet, $n=37 .{ }^{*} p<0.05$ between $\mathrm{h}-U C P 3$ and WT mice using an ANOVA statistical test for three repeated parameters

increase in body weight compared with h-UCP3 mice. The weight gain of h-UCP3 mice fed a high-fat diet did not differ from that of WT and h-UCP3 mice fed a chow diet. These observations were made in both male and female mice. There was no difference in linear growth as assessed by the length of the femur (data not shown). Food intake recordings did not reveal any difference between h-UCP3 and WT mice (data not shown). The difference observed in body weight between h-UCP3 and WT male mice fed highfat diet resulted from different fat depot weights (Fig. 6). A similar tendency was observed in female mice (pool of the four main white fat depots: $8.9 \pm 0.1 \mathrm{~g}$ vs $10.7 \pm 0.2 \mathrm{~g}$ in h-UCP3 and WT mice, $n=6, p<0.1)$. No difference was observed in weights of skeletal muscles, of brown adipose tissue and of various organs that do not express UCP3 (brain, liver and spleen).

Glucose and insulin tolerance tests in h-UCP3 mice Glucose tolerance tests were performed on male and female mice after 10 and 14 weeks of high-fat diet. Insulin tolerance tests were performed after 22 weeks of high-fat feeding. No difference in glucose tolerance tests was 
a
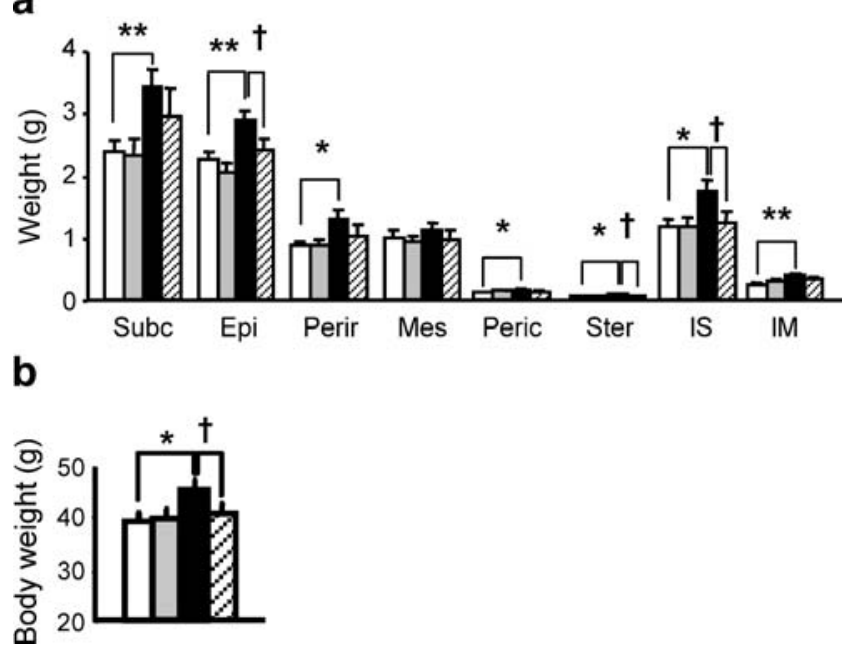

Fig. 6 a Weights of various white fat deposits from male mice after 23 weeks of diet. Open bars, WT fed a chow diet $(n=10)$; grey bars, h-UCP3 mice fed a chow diet $(n=12)$; filled bars, WT fed a high-fat diet $(n=10)$; hatched bars, h-UCP3 mice fed a high-fat diet $(n=10)$. Subc, subcutaneous; Epi, epididymal; Perir, perirenal; Mes, mesenteric; Peric, pericardic; Ster, sternal; $I S$, interscapular; $I M$, intermuscular. b Weights of male mice at the end of protocol. ${ }^{*} p<0.05$ and $* * p<0.01$ for differences between WT mice on chow and high-fat diets; $\dagger, p<0.05$ for differences between WT and h-UCP3 mice fed a high-fat diet using a Mann-Whitney non-parametrical statistical test

observed between genotypes 10 weeks after weaning (data not shown). At 14 weeks of high-fat feeding, the response differed according to the sex of the mice. When female mice were matched for body weight, h-UCP3 mice $(27 \pm 2 \mathrm{~g})$ were more glucose-tolerant than WT mice $(30 \pm 3 \mathrm{~g}$; Fig. 7a, b). No difference was seen in insulin tolerance test (Fig. 7c). Conversely, no difference in glucose tolerance tests was observed in male mice at 14 weeks of high-fat feeding (Fig. 8a). However, h-UCP3 mice weighed less than WT mice $(34 \pm 2 \mathrm{~g}$ vs $37 \pm 1 \mathrm{~g}, p<0.05)$ suggesting a possible impairment of glucose tolerance in $\mathrm{h}-U C P 3$ animals. The large sample size allowed subgrouping of animals by weight (Fig. 8c). h-UCP3 and WT male mice weighing less than $35 \mathrm{~g}$ showed superimposable blood glucose curves. In contrast, h-UCP3 male mice weighing more than $35 \mathrm{~g}$ were less glucose-tolerant than WT animals. Similarly, no difference was seen in insulin tolerance tests when all male mice were considered (Fig. 8b). Categorised by weight, the heavier h-UCP3 male showed impaired insulin action compared with WT animals (Fig. 8d).

$\mathrm{VO}_{2}$ and $\mathrm{RQ}$ in $h$-UCP3 mice $\mathrm{h}-U C P 3$ or WT male and female mice were isolated in a four-chamber oxylet system (Bioseb) and data were collected over a $24 \mathrm{~h}$ period. Energy expenditure and RQ were determined in animals fed high-fat and chow diets for 19 weeks. Energy expenditure did not
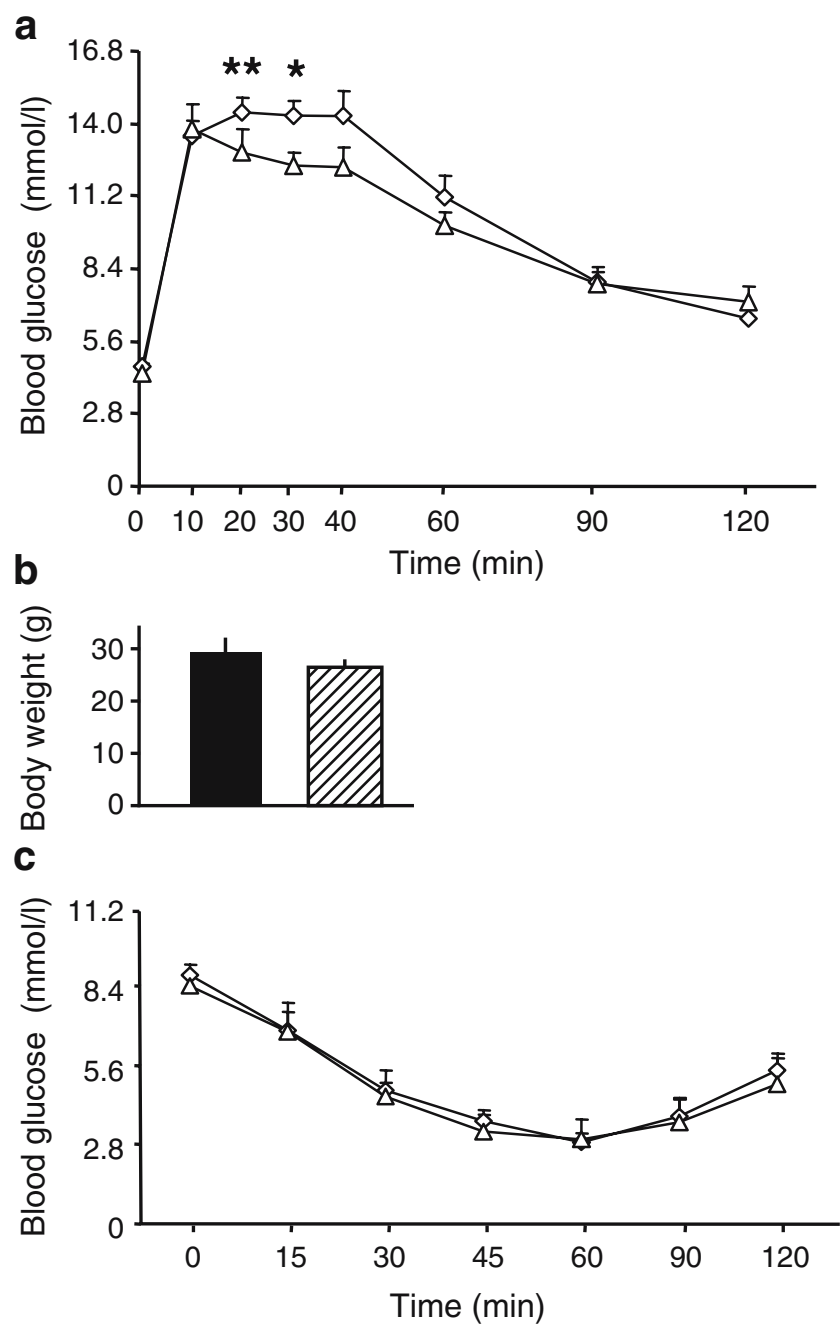

Fig. 7 Glucose and insulin tolerance tests in female mice on high-fat diet. a Glucose tolerance test was performed after 14 weeks on high-fat diet. b Body weights at the time of the test. Filled column, WT $(n=9)$; hatched column, $\mathrm{h}-U C P 3$ mice $(n=12)$. c Insulin tolerance test was performed after 22 weeks on high-fat diet. Diamonds, WT $(n=9)$; triangles, h-UCP3 mice $(n=12)$. ${ }^{*} p<0.05$ and ${ }^{*} p<0.01$ for difference between WT and h-UCP3 mice using an ANOVA statistical test for two repeated parameters

differ between the genotypes (data not shown). Interestingly, the difference in body weight between h-UCP3 and WT male mice during high-fat diet corresponded to $13 \mathrm{~kJ}^{-1 a y}{ }^{-1}$ $\mathrm{kg}^{-0.75}$. This value constitutes the detection limit of our apparatus. We cannot therefore rule out that minor changes in energy expenditure contribute to the difference in body weight between $\mathrm{h}-U C P 3$ and WT mice. A genotype effect appeared when analysing RQ in male mice (Fig. 9a). On chow diet, h-UCP3 mice showed lower RQ than WT mice. High-fat feeding led to a decrease in RQ in WT but not in $\mathrm{h}-U C P 3$ mice. Therefore, the $\mathrm{h}-U C P 3$ mice seem to be resistant to the shift in substrate use induced by a high-fat diet. When male mice under high-fat diet were divided into two subgroups according to weight, the difference in RQ 

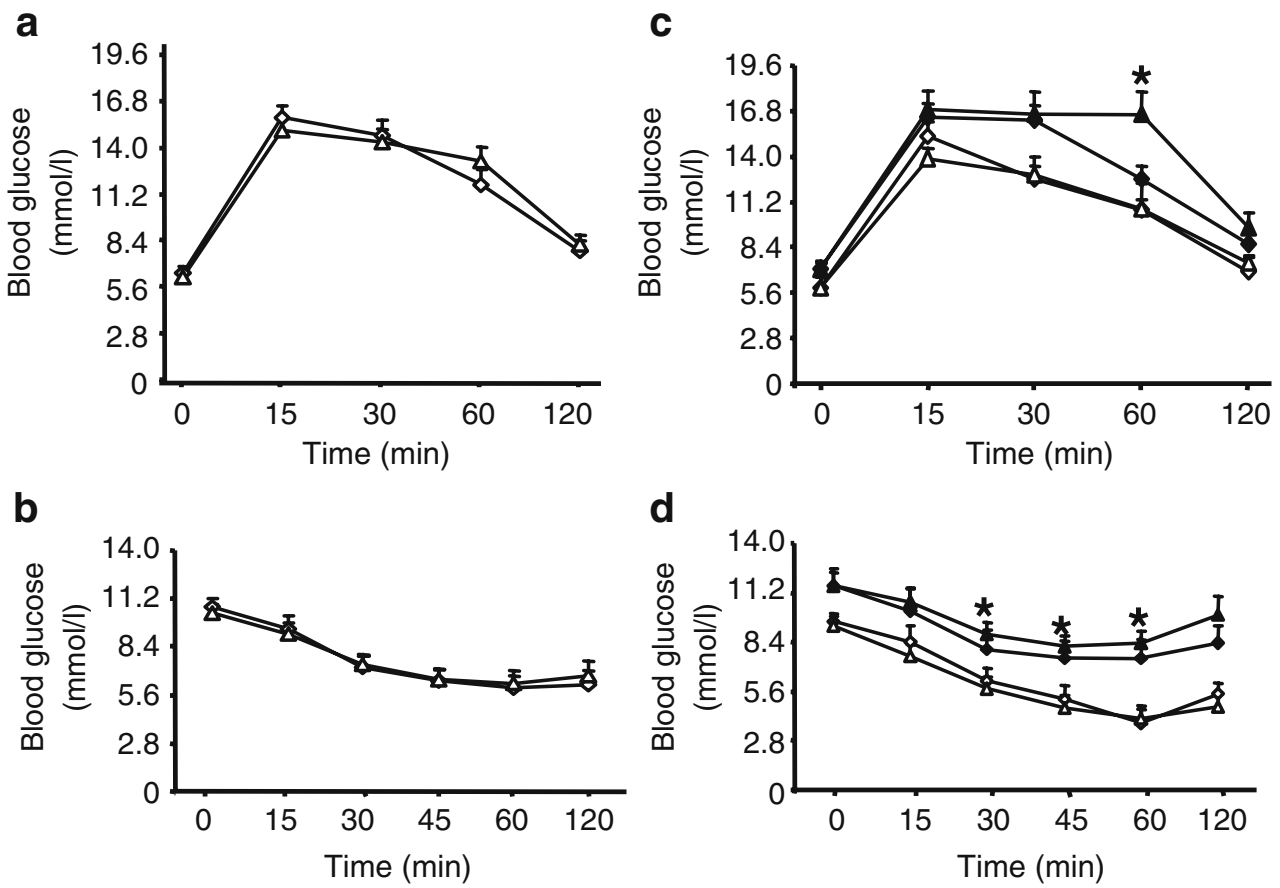

Fig. 8 Glucose and insulin tolerance tests in male mice on high-fat diet. Glucose (a) and insulin (b) tolerance tests were performed after 14 and 22 weeks on high-fat diet, respectively. Diamonds, WT, $n=30$ (a) and $n=26$ (b); triangles, h-UCP3 mice, $n=26$ (a) and $n=19$ (b). Animals were classified according to their body weights in glucose tolerance test (c) and insulin tolerance test (d). c open diamonds: WT, open triangles: h-UCP3 mice with body weights $<35 \mathrm{~g}(n=11$ and $n=14$,

between WT and h-UCP3 mice was more pronounced in the heavier animals (Fig. 9b,c). A difference in RQ was not observed in female mice (Fig. 9d).

\section{Discussion}

The aim of this study was to analyse the phenotype of a transgenic mouse model with a physiological overexpression of $U C P 3$ and with normal fibre-type specific distribution. The h-UCP3 transgene is under the control of its own regulatory elements. Therefore, overexpression is achieved in tissues expressing UCP3 in humans, i.e. in glycolytic and glycolytic-oxidative skeletal muscle, but not in tissues expressing UCP3 at higher levels in mice than in humans, i.e. brown adipose tissue. The rank order of expression, type IIB $\geq$ type IIA $>$ type I, is similar to the endogenous profile described in human skeletal muscles [5]. Previous transgenic models relied on heterologous promoters with activities in different fibre types and possibly in heart [27, 28]. Therefore, the increased expression of UCP3 specifically within glycolytic skeletal muscles is a major strength of the model. The second advantage is the magnitude of overexpression. UCP $3 \mathrm{mRNA}$ levels were increased from two- to sevenfold depending on the skeletal muscle

respectively); closed diamonds: WT; closed triangles: h-UCP3 mice with body weights $>35 \mathrm{~g}$ ( $n=19$ and $n=11$, respectively). d open symbols: as for c but with body weights $<45 \mathrm{~g}(n=14$ and $n=10$, respectively); filled symbols as for $\mathbf{c}$ but with body weights $>45 \mathrm{~g}$ ( $n=$ 12 and $n=9$, respectively). ${ }^{*} p<0.05$ between WT and h-UCP3 mice with heavy body weights using an ANOVA statistical test for three repeated parameters

analysed. In mitochondria from the gastrocnemius muscle, UCP3 protein levels are doubled in $\mathrm{h}-U C P 3$ mice compared with WT animals. The overexpression of $U C P 3$ is much lower than that of most of the previous models both in terms of mRNA (66- and 18-fold induction) and protein (25- and 15-fold induction) [27, 28]. Moreover, the range of expression in h-UCP3 mice is compatible with the inductions observed in vivo in humans during fasting and thyroid hormone or etomoxir treatments [19-21]. A recent study reported data with a twofold overexpression of $U C P 3$ in hindlimb muscle of $\mathrm{C} 57 \mathrm{Bl} / 6$ mice $[29,30]$. In this model, however, UCP3 expression was driven by the heterologous $\alpha$-skeletal actin promoter. As stated above, use of the $\mathrm{h}-U C P 3$ gene as the transgene is an obvious advantage of the model we used. It should, however, be pointed out that functional differences may exist between human and mouse UCP3, although none have been reported so far. The h-UCP3 mice therefore represent a good model for in vivo analysis of physiological UCP3 increase in skeletal muscle.

The moderate overexpression of $U C P 3$ led to an increase in mitochondrial oxygen consumption and reduced oxidative phosphorylation efficiency in permeabilised muscle fibres from glycolytic muscles. No effect was observed in an oxidative muscle that showed no difference in $U C P 3$ expression. Permeabilised muscle fibres offer the great 
a

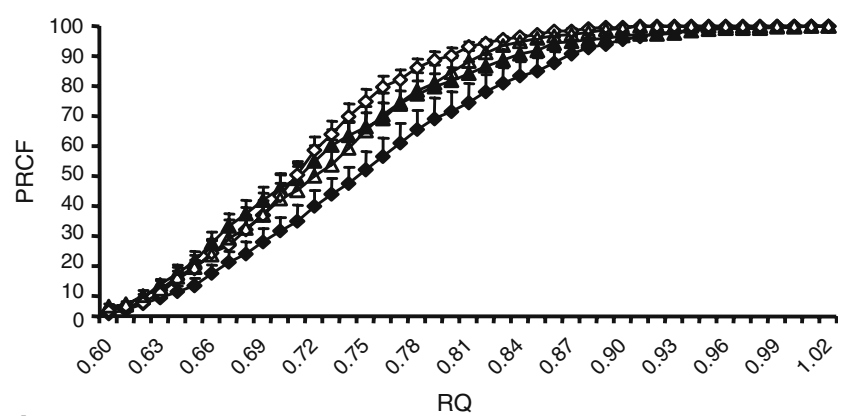

b

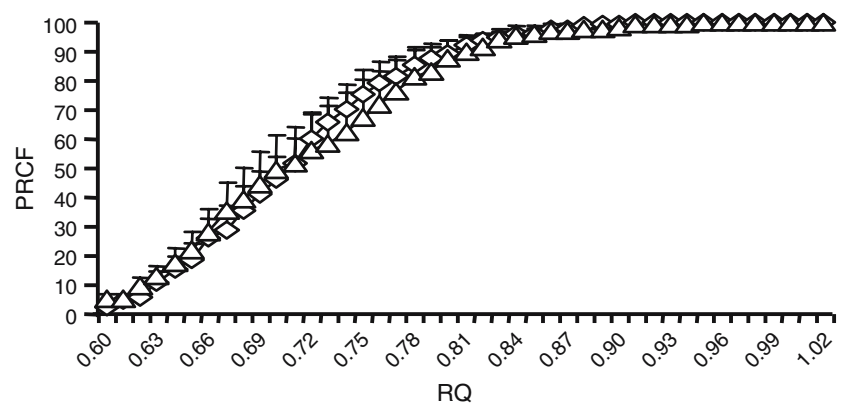

C

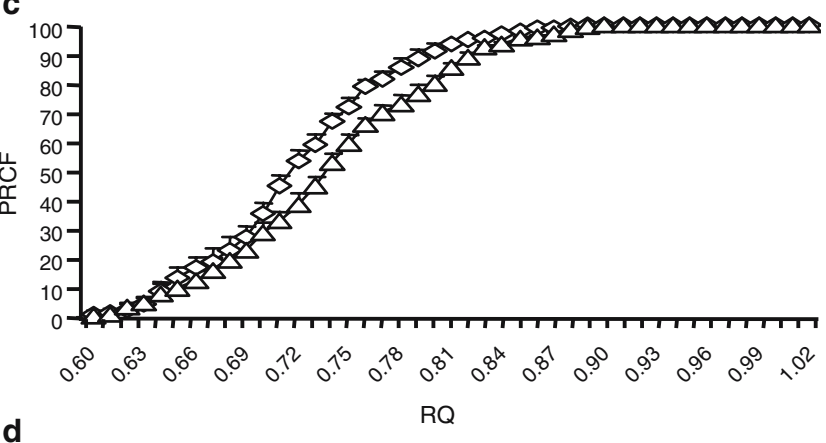

d

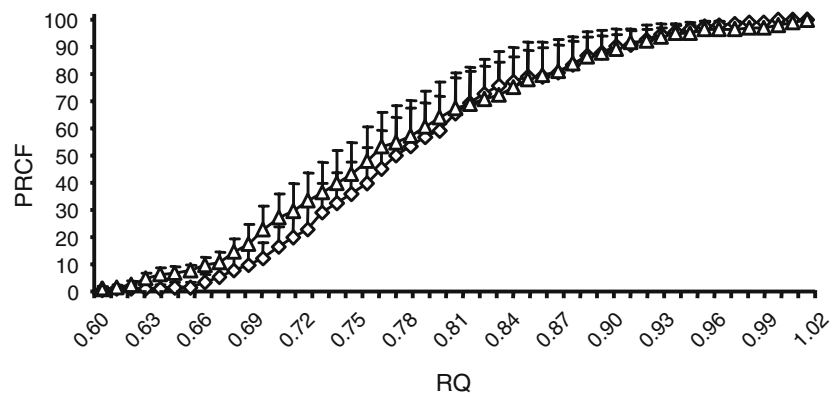

Fig. 9 Per cent relative cumulative frequency (PCRF) of RQ. a Male mice on chow or high-fat diet for 19 weeks. Filled diamonds: WT, filled triangles: h-UCP3 mice on chow diet ( $n=16$ in each group); open diamonds: WT, open triangles $\mathrm{h}-U C P 3$ mice on high-fat diet ( $n=11$ in each group). b, c, d Diamonds: WT; triangles: h-UCP3 mice. b Male mice on high-fat diet with body weights $<41 \mathrm{~g}(n=6$ in each group). c Male mice on high-fat diet with body weights $>41 \mathrm{~g}$ ( $n=5$ in each group). d Female mice on high-fat diet for 19 weeks $(n=$ 6 in each group)

advantage of being able to study mitochondria directly within the cellular context of the fibres, reducing the damage caused by isolation procedure [31]. Our data strongly suggest that a twofold increase in UCP3 levels enhances mitochondrial uncoupling in glycolytic muscles. Existing transgenic models support an uncoupling role of UCP3 [27, 28]. Studies of Ucp3 knockout mice have revealed reduced proton conductance and increased respiratory control ratio in skeletal muscle mitochondria $[6,7]$. However, these results have been challenged [32, 33]. Increased efficiency of cellular ATP production has been reported in $U c p 3$ knockout mice, suggesting an uncoupling by UCP3 in skeletal muscle in vivo [34]. As recently reviewed, several lines of evidence support the suggestion that UCP3 catalyses net proton conductance in the presence of activators [3, 4].

Body weight gain was similar in WT and h-UCP3 mice fed a chow diet. Accordingly, no difference was observed in $U c p 3$ knockout mice $[6,7,30]$. Recently, the effect of constitutive UCP3 overexpression at physiological levels on body weight and adiposity was reported [30]. After 4 and 8 months of low-fat diet, transgenic mice had lower body weights due to decreased adipose tissue mass. The effect was much less pronounced than that reported in the original transgenic model with high overexpression of UCP3 [27, 35]. Differences in diet composition, age, mouse strain $(\mathrm{C} 57 \mathrm{Bl} / 6$ vs $\mathrm{B} 6 \mathrm{D} 2$ background for h-UCP3 mice) and UCP3 expression profile ( $\alpha$-skeletal actin promoter vs endogenous human regulatory sequences) may explain the discrepancies between existing UCP3overexpressing transgenic models and $\mathrm{h}-U C P 3$ mice. However, h-UCP3 transgenic male or female mice on high-fat diet were leaner and had less white adipose tissue than WT littermates, suggesting that, under appropriate dietary challenge, the level of $U C P 3$ expression in glycolytic muscles controls adiposity. Numerous transgenic mouse models show resistance to an increased fat mass that is observed on high-fat diet but not on the standard chow diet [36]. However, most genetic manipulations concern genes that have a predominant function in adipose tissue. Few models in which skeletal muscle-specific overexpression of a transgene counteracts high-fat diet-induced obesity have been described. One such model is the transgenic mouse expressing glucokinase in skeletal muscle [37]. Interestingly, the increase of UCP3 mRNA levels in the skeletal muscle of these mice was suggested to mediate the prevention of diet-induced obesity. The phenotype of the $\mathrm{h}-U C P 3$ mice supports this hypothesis.

A clear sexual dimorphism appeared, when assessing glucose tolerance in h-UCP3 mice fed a high-fat diet. Despite lower body weight, h-UCP3 male mice had glucose tolerance similar to that of WT mice. When only the heavier animals were considered, the difference became significant, suggesting an impairment of glucose tolerance and insulin sensitivity in male mice overexpressing $U C P 3$. In contrast, analysis of weight-matched female mice revealed that $\mathrm{h}-U C P 3$ mice were more glucose-tolerant. 
Indirect calorimetry also revealed sexual dimorphism. $\mathrm{h}-U C P 3$ male mice had a lower RQ than WT mice on chow diet. On high-fat diet, the shift from carbohydrate to lipid use occurring in WT mice was totally blunted in h$U C P 3$ mice. Indeed, $\mathrm{h}-U C P 3$ mice displayed an $\mathrm{RQ}$ that did not differ when the animals were fed chow and high-fat diets. The difference between $\mathrm{h}-U C P 3$ and WT male mice was more pronounced in the heaviest animals. Altogether, these data suggest an impairment of metabolic flexibility in male h-UCP3 mice [38, 39]. An increase in UCP3 expression in skeletal muscle of male mice could diminish the ability to switch between glucose and fat oxidation. Indeed, the competition between glucose and fat oxidation resulting in metabolic inflexibility may occur within the mitochondria [40]. This inflexibility could be associated with deteriorated glucose tolerance. Interestingly, the adaptation of h-UCP3 mice at the level of substrate use and insulin sensitivity clearly differs from the effect on adiposity that was present in both sexes. Given the complexity of whole-body mechanisms determining glucose and fat oxidation, as well as glucose tolerance, there is no straightforward explanation of the sexual dimorphism observed in h-UCP3 mice. However, this is not surprising as in skeletal muscle more than one-half of the genes show differences of expression between male and female mice [41]. In fact, cis and trans regulation of gene expression appears to show a high degree of sex-dependence [42]. Several examples of sexual dimorphism have been reported in the literature including transgenic models relevant to metabolism, such as mice deficient in the peroxisomeproliferator-activated receptor $\alpha$ and apolipoprotein E [42, 43]. Study of the interplay between sex hormones and skeletal muscle metabolism merits further attention to elucidate the basis for the differences between male and female h-UCP3 mice.

In conclusion, we have created a novel transgenic mouse model with a physiological increase in UCP3 expression and a normal distribution between different skeletal muscle categories. Higher mitochondrial uncoupling was found in skeletal muscle from $\mathrm{h}-U C P 3$ mice. Due to decreased adipose tissue mass, the moderate overexpression of UCP3 results in leaner animals when animals are fed a high-fat diet. Sexual dimorphism was observed for glucose tolerance and whole-body substrate use. h-UCP3 mice may constitute a useful model for elucidation of the mechanisms determining metabolic flexibility in skeletal muscle.

Acknowledgements This work was supported by grants from Inserm (Progres 4P007E) and the Institut de Recherches Servier. We are very grateful to V. Bezaire (Obesity Research Laboratory Inserm U858) for critical reading of the manuscript and constructive criticisms. We thank L. Monbrun from the Animal Physiological Platform of the Toulouse Genopole and M.-A. Marques from the Obesity Research Laboratory Inserm U858. We also thank Y. Barreira and her team from the animal core facility of the Louis Bugnard Institute and F. Pillard from Obesity Research Laboratory Inserm U858.

Duality of interest The authors declare that there is no duality of interest associated with this manuscript.

\section{References}

1. Lowell BB, Shulman GI (2005) Mitochondrial dysfunction and type 2 diabetes. Science 307:384-387

2. Ricquier D, Bouillaud F (2000) The uncoupling protein homologues: UCP1, UCP2, UCP3, StUCP and AtUCP. Biochem J 345:161-179

3. Krauss S, Zhang CY, Lowell BB (2005) The mitochondrial uncoupling-protein homologues. Nat Rev Mol Cell Biol 6:248261

4. Brand MD, Esteves TC (2005) Physiological functions of the mitochondrial uncoupling proteins UCP2 and UCP3. Cell Metab 2:85-93

5. Hesselink MKC, Keizer HA, Borghouts LB et al (2001) Protein expression of UCP3 differs between human type 1, type 2a, and type 2 b fibers. FASEB J 15:1071-1073

6. Vidal-Puig AJ, Grujic D, Zhang C-Y et al (2000) Energy metabolism in uncoupling protein 3 gene knockout mice. J Biol Chem 275:16258-16266

7. Gong DW, Monemdjou S, Gavrilova O et al (2000) Lack of obesity and normal response to fasting and thyroid hormone in mice lacking uncoupling protein-3. J Biol Chem 275:1625116257

8. Barger JL, Barnes BM, Boyer BB (2006) Regulation of UCP1 and UCP3 in arctic ground squirrels and relation with mitochondrial proton leak. J Appl Physiol 101:339-347

9. Mills EM, Banks ML, Sprague JE, Finkel T (2003) Pharmacology: uncoupling the agony from ecstasy. Nature 426:403404

10. Brownlee M (2001) Biochemistry and molecular cell biology of diabetic complications. Nature 414:813-820

11. Du XL, Edelstein D, Dimmeler S, Ju Q, Sui C, Brownlee M (2001) Hyperglycaemia inhibits endothelial nitric oxide synthase activity by posttranslational modification at the Akt site. J Clin Invest 108:1341-1348

12. Brand MD, Pamplona R, Portero-Otin M et al (2002) Oxidative damage and phospholipid fatty acyl composition in skeletal muscle mitochondria from mice underexpressing or overexpressing uncoupling protein 3. Biochem J 368:597-603

13. Schrauwen P, Hoeks J, Schaart G et al (2003) Uncoupling protein 3 as a mitochondrial fatty acid anion exporter. FASEB J 17:2272-2274

14. Himms-Hagen J, Harper M-E (2001) Physiological role of UCP3 may be export of fatty acids from mitochondria when fatty acid oxidation predominates: an hypothesis. Exp Biol Med (Maywood) 226:78-84

15. MacLellan JD, Gerrits MF, Gowing A, Smith PJ, Wheeler MB, Harper ME (2005) Physiological increases in uncoupling protein 3 augment fatty acid oxidation and decrease reactive oxygen species production without uncoupling respiration in muscle cells. Diabetes 54:2343-2350

16. Mozo J, Ferry G, Studeny A et al (2006) Expression of UCP3 in $\mathrm{CHO}$ cells does not cause uncoupling, but controls mitochondrial activity in the presence of glucose. Biochem J 393:431439

17. Garcia-Martinez C, Sibille B, Solanes G et al (2001) Overexpression of UCP3 in cultured human muscle lowers mitochon- 
drial membrane potential, raises ATP/ADP ratio, and favors fatty acid versus glucose oxidation. FASEB J 15:2003-2035

18. Guigal N, Rodriguez M, Cooper RN et al (2002) Uncoupling protein-3 (UCP3) mRNA expression in reconstituted human muscle after myoblast transplantation in RAG2-/-/gamma c/C5immunodeficient mice. J Biol Chem 277:47407-47411

19. Millet L, Vidal H, Andreelli $F$ et al (1997) Increased uncoupling protein-2 and -3 mRNA expression during fasting in obese and lean humans. J Clin Invest 100:2665-2670

20. Barbe P, Larrouy D, Boulanger C et al (2001) Triiodothyroninemediated upregulation of UCP2 and UCP3 gene expression in human skeletal muscle without coordinated induction of mitochondrial respiratory chain genes. FASEB J 15:13-15

21. Schrauwen P, Hinderling V, Hesselink MKC et al (2002) Etomoxirinduced increase in UCP3 supports a role of uncoupling protein 3 as a mitochondrial fatty acid anion exporter. FASEB J 16:1688-1690

22. Lucas S, Tavernier G, Tiraby C, Mairal A, Langin D (2003) Expression of human hormone-sensitive lipase in white adipose tissue of transgenic mice increases lipase activity but does not enhance in vitro lipolysis. J Lipid Res 44:154-163

23. Tavernier G, Toumaniantz G, Erfanian M et al (2003) B3Adrenergic stimulation produces a decrease of cardiac contractility ex vivo in mice overexpressing the human $\beta 3$-adrenergic receptor. Cardiovasc Res 59:288-296

24. Appleyard SM, Hayward M, Young JI et al (2003) A role of endogenous opioid beta-endorphin in energy homeostasis. Endocrinology 144:1753-1760

25. Jimenez M, Yvon C, Lehr L et al (2002) Expression of uncoupling protein-3 in subsarcolemmal and intermyofibrillar mitochondria of various mouse muscle types and its modulation by fasting. Eur J Biochem 269:2878-2884

26. Riachi M, Himms-Hagen J, Harper ME (2004) Percent relative cumulative frequency analysis in indirect calorimetry: application to studies of transgenic mice. Can J Physiol Pharmacol 82:10751083

27. Clapham JC, Arch JRS, Chapman H et al (2000) Mice overexpressing human uncoupling protein-3 in skeletal muscle are hyperphagic and lean. Nature 406:415-418

28. Son C, Hosoda K, Ishihara K et al (2004) Reduction of dietinduced obesity in transgenic mice overexpressing uncoupling protein 3 in skeletal muscle. Diabetologia 47:47-54

29. Bezaire V, Spriet LL, Campbell S et al (2005) Constitutive UCP3 overexpression at physiological levels increases mouse skeletal muscle capacity for fatty acid transport and oxidation. FASEB J 19:977-979

30. Costford SR, Chaudhry SN, Salkhordeh M, Harper ME (2006) Effects of the presence, absence, and overexpression of uncou- pling protein-3 on adiposity and fuel metabolism in congenic mice. Am J Physiol Endocrinol Metab 290:E1304-E1312

31. Saks VA, Veksler VI, Kuznetsov AV et al (1998) Permeabilized cell and skinned fiber techniques in studies of mitochondrial function in vivo. Mol Cell Biochem 184:81-100

32. Bezaire V, Hofmann W, Kramer JKG, Kozak LP, Harper M-E (2001) Effects of fasting on muscle mitochondrial energetics and fatty acid metabolism in Ucp3(-/-) and wild-type mice. Am J Physiol Endocrinol Metab 281:E975-E982

33. Cadenas S, Echtay KS, Harper JA et al (2002) The basal proton conductance of skeletal muscle mitochondria from transgenic mice overexpressing or lacking uncoupling protein-3. J Biol Chem 277:2773-2778

34. Cline GW, Vidal-Puig AJ, Dufour S, Cadman KS, Lowell BB, Shulman GI (2001) In vivo effects of uncoupling protein-3 gene disruption on mitochondrial energy metabolism. J Biol Chem 276:20240-20244

35. Changani KK, Nicholson A, White A, Latcham JK, Reid DG, Clapham JC (2003) A longitudinal magnetic resonance imaging (MRI) study of differences in abdominal fat distribution between normal, and lean overexpressers of mitochondrial uncoupling-3 (UCP-3). Diab Obes Metab 5:99-105

36. Valet P, Tavernier G, Castan-Laurell I, Saulnier-Blache JS, Langin D (2002) Understanding adipose tissue development from transgenic animal models. J Lipid Res 43:835-860

37. Otaegui PJ, Ferre T, Riu E, Bosch F (2003) Prevention of obesity and insulin resistance by glucokinase expression in skeletal muscle of transgenic mice. FASEB J 17:2097-2099

38. Storlien L, Oakes ND, Kelley DE (2004) Metabolic flexibility. Proc Nutr Soc 63:363-368

39. Kelley DE (2005) Skeletal muscle fat oxidation: timing and flexibility are everything. J Clin Invest 115:1699-1702

40. Ukropcova B, McNeil M, Sereda O et al (2005) Dynamic changes in fat oxidation in human primary myocytes mirror metabolic characteristics of the donor. J Clin Invest 115:19341941

41. Yang X, Schadt EE, Wang S et al (2006) Tissue-specific expression and regulation of sexually dimorphic genes in mice. Genome Res 16:995-1004

42. Wang S, Yehya N, Schadt EE, Wang H, Drake TA, Lusis AJ (2006) Genetic and genomic analysis of a fat mass trait with complex inheritance reveals marked sex specificity. PLoS Genet 2:e15

43. Costet P, Legendre C, More J, Edgar A, Galtier P, Pineau T (1998) Peroxisome proliferator-activated receptor $\alpha$-isoform deficiency leads to progressive dyslipidemia with sexually dimorphic obesity and steatosis. J Biol Chem 273:29577-29585 